\title{
The role of serology in active ocular toxoplasmosis
}

\author{
Marina Papadia $\cdot$ Raffaella Aldigeri • \\ Carl P. Herbort
}

Received: 24 August 2011/Accepted: 22 December 2011/Published online: 11 January 2012

(C) Springer Science+Business Media B.V. 2012

\begin{abstract}
Our purpose was to examine toxoplasmic serology in relation to episodes of suspected acute toxoplasmic retinochoroiditis and evaluate its use in the appraisal of patients. The mean values of enzymatic immunoassay (EIA) titers for toxoplasmic antibodies were retrospectively compared in patients with active and inactive toxoplasmosis and in a third group of uveitis cases not caused by toxoplasmosis. The proportion of cases under and above a predefined serology value above cut-off was compared in all groups. Between 1995 and 2010, 97 out of 1,276 new uveitis cases seen at the Centre for Ophthalmic Specialized Care (COS), Lausanne, Switzerland were diagnosed as toxoplasmic retinochoroiditis, of which 51 had documented serology available. The mean EIA
\end{abstract}

M. Papadia $(\square)$. C. P. Herbort $(\square)$

Centre for Ophthalmic Specialized Care (COS),

6, rue de la Grotte, 1003 Lausanne, Switzerland

e-mail: marinapapadia@yahoo.com

C. P. Herbort

e-mail: carl.herb@bluewin.ch

M. Papadia

Eye Clinic, Department of Neurosciences, Ophthalmology and Genetics, University of Genova, Genova, Italy

R. Aldigeri

Head and Neck Department, Section of Ophthalmology,

University of Parma, Parma, Italy

C. P. Herbort

University of Lausanne, Lausanne, Switzerland values for immunoglobulin (Ig) $\mathrm{G}$ were $147.75 \pm$ $259.4 \mathrm{IU} / \mathrm{ml}$ for patients with active disease, $18.93 \pm$ $23.09(p<0.05)$ for patients with inactive toxoplasmosis and $18.35 \pm 20.82$ for controls $(p<0.017)$. The proportion of cases under the designated limit value were $2 / 51(4 \%)$ in the active retinitinochoroiditis group, 14/27 $(52 \%)(p<0.05)$ in the control group, and $7 / 7(100 \%)$ in the inactive toxoplasmic group $(p<0.001)$. Three out of 51 cases showed high IgM values in addition to IgG elevation and were primary infections. Toxoplasmosis serology, contrary to popular belief, is useful to confirm active toxoplasmic retinochoroiditis; it is easy to perform, cheap and supports clinical diagnosis in up to $96 \%$ of cases, not only by showing positivity but by also showing a significant elevation of titers. In atypical cases serology is not only useful but essential.

Keywords Serology · Toxoplasmic retinochoroiditis $\cdot$ Active disease $\cdot$ Atypical cases

\section{Introduction}

The usefulness of toxoplasmosis serology is very often downplayed in textbooks and articles on the topic, and at uveitis symposia, being mostly regarded as unnecessary [1]. In most cases toxoplasmic retinochoroiditis is a recurrence of a previous systemic infection and only positive for immunoglobulin (Ig) G [2]. Serology is nevertheless essential to establish previous infection 
in order to be able to attribute the disease to this agent, even though it is said that the diagnosis is mainly clinical [3]. It has often been noted that the increase in IgG anti-toxoplasmic titers is associated with recurrent disease [4]. This shows that in toxoplasmic retinochoroiditis, even when it presents as the first apparent ocular episode, a silent systemic primo-infection has occurred previously with the clandestine establishment of cysts in the retina [5]. It has to be emphasized, contrary to all publications published before the 1990s, that absence of IgM does not indicate that we are dealing with a congenital disease, but that most cases of toxoplasmic retinochoroiditis are due to acquired toxoplasmosis following a previous undetected primary infection $[6,7]$.

In this study we wanted to explore toxoplasmic serologies in episodes of acute toxoplasmic retinochoroiditis, in patients with an inactive toxoplasmic scar and in a control group of uveitis patients.

\section{Materials and methods}

We retrospectively reviewed 1,276 charts of uveitis patients that presented to the Centre for Ophthalmic Specialized Care (COS), Lausanne, Switzerland between 1995 and 2010.

All research followed the tenets of the declaration of Helsinki.

The aim of the study was to analyze serological data of patients with active retinochoroiditis and compare them to a group of patients with inactive ocular toxoplasmosis and a group of controls. The control group consisted of other uveitis patients in whom toxoplasmic serology had been performed as part of a general work-up and found to be positive, but where toxoplasmic etiology of the ocular disease could be ruled out on the basis of the clinical appearance of the disease.

For this purpose, the mean IgG titers of enzymatic immunoassay (EIA) for toxoplasmic antibodies were compared in the three groups and, furthermore, the proportion of cases under and above a value of serology fixed at four times or at twenty times the cut-off value was compared in the active and inactive toxoplasmosis groups and in the control group.

EIA was performed to obtain toxoplasmic serology. EIA is a biochemical technique used mainly in immunology to detect the presence of an antibody or an antigen in a sample. In this setting it was used to detect and quantify an unknown amount of antitoxoplasmic antibodies taken from the serum of a patient. In brief, serum from a patient with an unknown amount of a specific antibody is poured into a recipient coated with the corresponding antigen. The antibody will bind to the antigen and create a complex. An antibody to IgG linked to an enzyme is then washed over the surface so that it can bind to the complex. In the final step a substance is added to enable the fixed enzyme to convert to a detectable signal. Thus, when a light of the appropriate wavelength is shone upon the sample, the antigen/antibody complexes will fluoresce so that the amount of antibody in the sample can be inferred through the magnitude of the fluorescence.

Data entry and statistical analysis were performed using SPSS version 17.0 software. ANOVA was used to compare the mean values of toxoplasmosis antibodies among groups. Differences in proportions of high levels of antibodies were evaluated by Pearson's Chi-squared test. A statistical test result was considered significant with a $p$ value of $<0.05$.

\section{Results}

Ninety-seven out of 1,276 new uveitis patients seen at Centre for Ophthalmic Specialized Care (COS), Lausanne, Switzerland between 1995 and 2010 were found to be affected by toxoplasmosis retinochoroiditis on the basis of clinical signs.

The demographic data of the three groups are shown in Table 1.

Eighty-five cases had active disease and 51 had documented serology for Toxoplasma gondii. All these patients had either a retinochoroidal focus $(n=49)$ or a papillitis $(n=2)$. In one third of patients $(n=16,31 \%)$ a focus without a juxtalesional scar was found, indicating that the proportion of cases with a so-called 'typical' presentation was no more than two-thirds. Table 2 summarizes the results of serology in the three groups.

The mean EIA values were $147.75 \pm 259.4$ international units $(\mathrm{IU} / \mathrm{ml})$ in patients with active retinochoroiditis $(n=51), \quad 18.35 \pm 20.82 \mathrm{IU} / \mathrm{ml}$ in controls $(n=27)(p<0.05)$, and $18.93 \pm 23.09 \mathrm{IU} /$ $\mathrm{ml}$ in patients with inactive toxoplasmosis $(n=7)$ $(p<0.017)$ (Fig. 1). A post hoc analysis confirmed a significant difference in the mean EIA values between 
Table 1 Demographic data of the patients

\begin{tabular}{llll}
\hline & $\begin{array}{l}\text { Patients with active } \\
\text { retinochoroiditis }(n=51)\end{array}$ & Controls $(n=27)$ & $\begin{array}{l}\text { Patients with inactive } \\
\text { retinochoroiditis }(n=7)\end{array}$ \\
\hline Sex ratio $(\mathrm{M} / \mathrm{F})$ & $22 / 29$ & $11 / 16$ & $4 / 3$ \\
Mean age $\pm \mathrm{SD}$ & $36.55 \pm 17.54$ & $50.78 \pm 17.34$ & $34.67 \pm 19.32$ \\
\hline
\end{tabular}

Table 2 Data on serological test

\begin{tabular}{llll}
\hline & $\begin{array}{l}\text { Active } \\
\text { disease } \\
(n=51)\end{array}$ & $\begin{array}{l}\text { Control } \\
(n=27)\end{array}$ & $\begin{array}{l}\text { Inactive } \\
\text { disease } \\
(n=7)\end{array}$ \\
\hline $\begin{array}{c}\text { Serological test (4 times } \\
\text { cut-off value of anti- } \\
\text { toxoplasma IgG titer) }\end{array}$ & 49 & 13 & 0 \\
$\begin{array}{c}\text { Serological test (20 times } \\
\text { cut-off value of anti- } \\
\text { toxoplasma IgG titer) }\end{array}$ & 23 & 2 & 0 \\
\hline
\end{tabular}

patients with active retinochoroiditis and the control group (Bonferroni method, $p<0.05$ ).

Forty-nine patients $(96 \%)$ had titers above the chosen limit considered as an elevated titer (Table 2). Three patients also presented a high IgM titer indicating a primary ocular infection. In the inactive toxoplasmosis group 0/7 patients (false positives) had elevated titers and in the control group 13/27 (48\%) patients (false positives) had elevated titers (Table 2).

The high number of false positives in the control group of uveitis patients is most probably explained by the fact that the proportion of cases of sarcoidosis in this group was high. It is well known that sarcoidosis patients present a rise of antibodies against latent infections [8].

When the defined serology value was fixed at 20 times cut-off value, 23/51 (45\%) patients with active disease were positive and only $2 / 27$ (7\%) controls were positive, and none was positive in the inactive cases.

Chi-squared test confirmed a significant association between high levels of toxoplasmosis antibodies and the presence of active retinochoroiditis $(p<0.001)$. Based on these data, the EIA test was very sensitive (49/51 positive tests in the presence of disease, sensitivity $=96 \%)$ and less specific $(13 / 27$ negative tests in the absence of disease, specificity $=48 \%$ ) when the defined value was four times the cut-off value; however, it was more specific (25/27 true negatives, specificity $=93 \%$ ) when the defined value was 20 times the cut-off value.

Figure 1 shows graphically the comparison of mean EIA titers for toxoplasmic antibodies in patients with active and inactive toxoplasmosis and in a third group of uveitis cases (control group) (Fig. 2).
Fig. 1 Comparison of mean EIA titers for toxoplasmic antibodies in patients with active and inactive toxoplasmosis and in a third group of uveitis cases (control group)

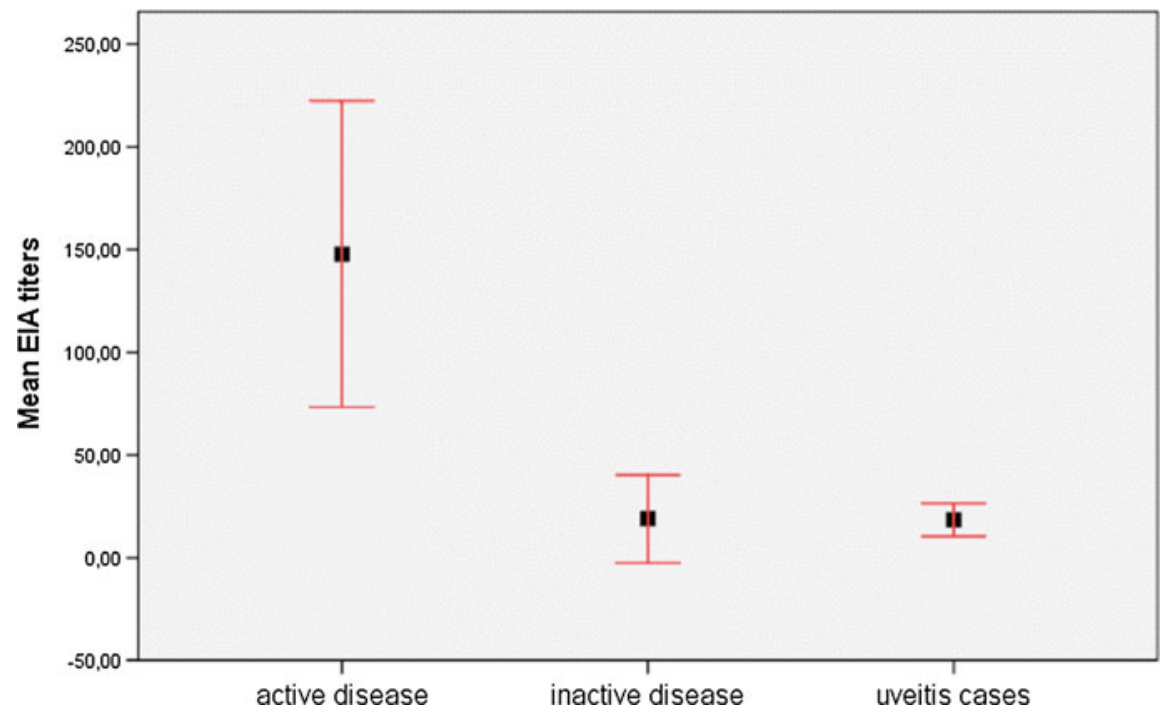




\section{Discussion}

To date, in the literature and at uveitis conferences the role of toxoplasmic serology in toxoplasmic retinochoroiditis has been downplayed and even considered irrelevant. This comes from the fact that general serology laboratories where samples from ocular patients are tested, do not understand the exact purpose of requesting a serology workup in a patient with ocular toxoplasmosis. They invariably respond that the serology shows an old toxoplasmic infection and is negative for an acute infection (no $\operatorname{IgM}$ ). This is an adequate response for internal medicine patients where an acute infection is usually searched for and motivates the serology quest. However, in ocular patients where, in the overwhelming majority of cases, the disease is the result of a recurrence, we need to know whether a clinical picture compatible with ocular toxoplasmosis is actually due to T. gondii and we therefore need to know if the patient has ever been in contact with the agent; this is confirmed by the presence of even low levels of IgG. Our study shows that in active cases of toxoplasmic retinochoroiditis, serology can be more rewarding. In 96 of 100 patients with an active uveitis compatible with ocular toxoplasmosis, a significant elevation of IgG titers (4 times the cut-off value) was found, indicating that serology is very sensitive and reasonably specific when compared to the control group (uveitis cases). If we consider titers 20 times higher than the cut-off value (very high titers) the specificity rises to $93 \%$.

When a clinician is dealing with suspected toxoplasmic retinochoroiditis, serology will not only be positive, showing that the patient has at one point been in contact with the agent, but there is also a $96 \%$ chance of finding an elevated IgG titer, making serology very sensitive in confirming a suspected diagnosis. Moreover if titers are very high (20 times cut-off value), serology is not only very sensitive but also very specific. A positive value in this situation means that the case can only be ocular toxoplasmosis. Such laboratory information is not to be neglected in typical cases but is essential in so-called atypical cases.

In summary, serology is useful in all active cases of suspected toxoplasmic retinochoroiditis. Very high titers are specific for toxoplasmic disease, thus confirming the suspected diagnosis. This is very important, especially in atypical cases.
Fig. 2 Column graph indicating the discriminatory power of serology

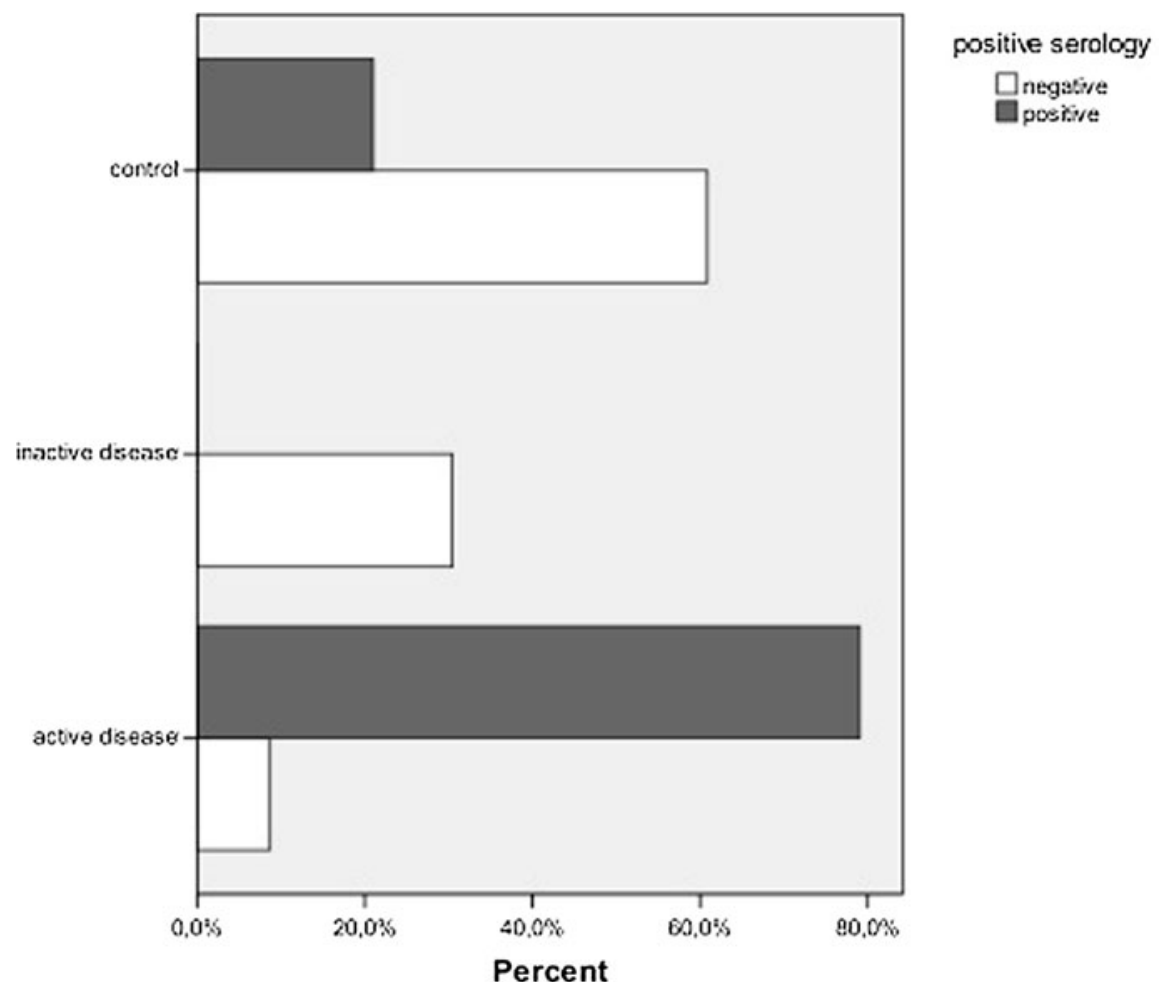




\section{References}

1. Commodaro AG, Belfort RN, Rizzo LV et al (2009) Ocular toxoplasmosis: an update and review of the literature. Mem Inst Oswaldo Cruz 104(2):345-350

2. Munio JC, Juarez CP, Luna JD et al (1999) The importance of specific IgG and IgE autoantibodies to retinal $\mathrm{S}$ antigen, total serum IgE, and sCD23 levels in autoimmune and infectious uveitis. J Clin Immunol 19(4):215-222

3. Pleyer U, Torun N, Liesenfield O (2007) Ocular toxoplasmosis. Ophthalmologe 104(7):603-615

4. Ongkosuwito JV, Bosch-Driessen EH, Kijlstra A, Rothova A (1999) Serologic evaluation of patients with primary and recurrent ocular toxoplasmosis for evidence of recent infection. Am J Ophthalmol 128(4):407-412

5. Holland GN (1999) Reconsidering the pathogenesis of ocular toxoplasmosis. Am J Ophthalmol 128(4):502-505

6. Silveira C, Belfort R Jr, Burnier M Jr et al (1998) Acquired toxoplasmic infection as the cause of toxoplasmic retinochoroiditis in families. Am J Ophthalmol 106(3):362-364

7. Bonfioli AA, Orefice F (2005) Toxoplasmois. Semin Ophthalmol 20(3):129-141

8. Berthoud Kündig JF, Keller A, Herbort CP (1994) Increase in polyclonal immunoglobulins: a possible useful aid in diagnosis of uveitis caused by sarcoidosis. Klin Monbl Augenheilkd 204(5):323-329 